levels were observed in the spleen when radio-resistant cells lack TLR4.

Conclusion These data suggest that TLR4 plays a role on both the bone marrow derived and local resident cells in aggravating experimental arthritis. TLR4 plays a role locally on the synovial fibroblasts by creating a more aggressive inflammatory environment in the joint cavity and thereby increasing joint destruction. Conversely, TLR4 activation on the bone marrow derived cells could increase $T$ cell activation by antigen presenting cells and thereby promoting a more aggressive Th17 phenotype and increase joint swelling.

\section{A97 IDENTIFYING THE TLR4 BEARING TARGET CELL IN EXPERIMENTAL ARTHRITIS}

Ben T van den Brand,' Shahla Abdollahi-Roodsaz,' Miranda B Bennink,' Onno J Arntz, Wim B van den Berg, ${ }^{1}$ Fons A J van de Loo' ${ }^{1}$ Department of Rheumatology, Nijmegen Centre for Molecular Life Sciences, Radboud University Nijmegen Medical Centre, Nijmegen, The Netherlands

\subsection{6/ard.2010.148973.15}

Purpose The interleukin 1 (IL-1) receptor antagonist (IL-1Ra) knockout mice spontaneously develop $\mathrm{T}$ cell driven arthritis due to excessive IL-1 signalling. Joint destruction is accompanied by increased Th17 cells and elevated IL-17 levels compared to wild type (Balb/c) mice. When cross bred with toll-like receptor 4 (TLR4) knock out $\left(\right.$ TLR $^{-{ }^{-}}{ }^{-}$) these animals showed reduced inflammation, joint destruction and diminished IL-17 levels. To reduce adverse effects of TLR4 inhibition a cell specific targeted therapy of arthritis is desired. Therefore, the authors set out to identify the TLR4 bearing cells in experimental arthritis responsible for increased IL-17 production and arthritis severity.

Method A reciprocal sex-mismatched bone marrow transplantation was performed with $\mathrm{TLR} 4^{-/-}$and TLR4 ${ }^{+/+}$mice in the IL-1 $\mathrm{Ra}^{-/-}$background and $\mathrm{Balb} / \mathrm{c}$ bone marrow as control. Y-chromosome staining of bone marrow was performed to assess engraftment. Clinical manifestation of disease was assessed macroscopically over time. Spleen and lymph node cells were isolated and subjected to $T$ helper subset analysis.

Results Engraftment of bone marrow was near 100\% successful as determined by Y-chromosome staining of the bone marrow, which indicates a successful reconstitution. Lack of TLR4 on either the engrafted bone marrow cells or the radioresistant cells in the joint did not affect disease incidence. However, animals that lacked TLR4 on the engrafted bone marrow derived cells, radio-resistant cells, or both showed reduced macroscopic arthritis scores. In mesenteric lymph nodes there were no differences observed in percentage of interferon $\gamma$ and IL-17 producing cells. Neither was there a difference in Th1 cells in the spleen. However, decreased Th17 